\title{
Mesure de la perméabilité des argiles sous contrainte et température
}

M. KHEMISSA ${ }^{(1)}$

Groupement pour l'étude des Structures Souterraines de Stockage (G.3S),

LMS (URA 317-CNRS),

École polytechnique,

91128 Palaiseau Cedex
Cet article présente les résultats d'essais réalisés au moyen d'un appareil développé au G.3S pour l'étude de la perméabilité des argiles sous contrainte et température imposées. Les essais ont été réalisés sur une éprouvette d'argile prélevée à $30 \mathrm{~m}$ de profondeur sur le site de Provins. Cette éprouvette a été soumise à différents gradients hydrauliques sous diverses températures. Les résultats des mesures mettent en évidence l'influence de la température sur la perméabilité de l'argile et montrent que cette influence est due à des variations de la viscosité de l'eau interstitielle. Ces résultats semblent toutefois indiquer que la perméabilité est peu sensible aux variations du gradient hydraulique.

Mots clés : argile, coefficient de perméabilité, viscosité, température, gradient hydraulique.

\section{Measurement of the permeability of clays under stress and temperature}

This paper present the tests results performed by means of an apparatus designed in G.3S to study the permeability of clays under imposed stress and temperature. The tests were carried out on a clay specimen taken from a depth of $30 \mathrm{~m}$ on the site of Provins. This specimen was subjected to several hydraulic gradients at various temperatures. The results highlight the influence of temperature on the permeability of clay and show that this influence is due to variations of the pore water viscosity. However, these results seem to indicate that the permeability is slightly sensitive to variations of hydraulic gradient.

Key words : clay, coefficient of permeability, viscosity. temperature, hydraulic gradient. 


\section{Introduction}

Les argiles comptent parmi les formations géologiques envisageables pour le stockage des déchets radioactifs qui dégagent de la chaleur, notamment à grandes profondeurs, oủ les massifs argileux sont généralement stables et peuvent atteindre plusieurs centaines de mètres d'épaisseur. Ces formations font donc l'objet de recherches théoriques et expérimentales, in situ et en laboratoire, comme celles menées actuellement au Groupement pour l'étude des Structures Souterraines de Stockage (G.3S, École polytechnique) dans le cadre général de l'étude des couplages thermo-hydro-mécaniques des milieux poreux saturés.

Les propriétés mécaniques et hydrauliques des argiles conditionnent le comportement des ouvrages de stockage et leur sûreté : la perméabilité est l'un des paramètres physiques dimensionnants. Celle-ci doit être très faible pour que les éventuels mouvements de l'eau interstitielle, créés par des gradients hydrauliques et thermiques, susceptibles d'entrainer les radionucléides vers la surface, soient maitrisés durant toute la période de leur activité.

Les méthodes expérimentales classiques utilisant des systèmes conventionnels (perméamètres à charge constante ou variables, œdomètre, triaxial) ne sont pas adaptêes à l'étude des propriétés hydrauliques des argiles profondes peu perméables sous contrainte et température en raison de l'extrême faiblesse des débits d'eau mis en jeu. La construction d'un appareillage spécifique permettant de mesurer de très faibles débits d'eau sous l'effet d'un gradient de pression interstitielle (i.e. gradient hydraulique) en fonction de la température et de la contrainte de confinement exercées, s'est donc avérée indispensable.

On décrit dans cet article la configuration du dispositif expérimental et le fonctionnement de chacun des éléments qui le composent. On expose ensuite le programme expérimental réalisé et la procédure d'essais suivie, puis on analyse les résultats obtenus.

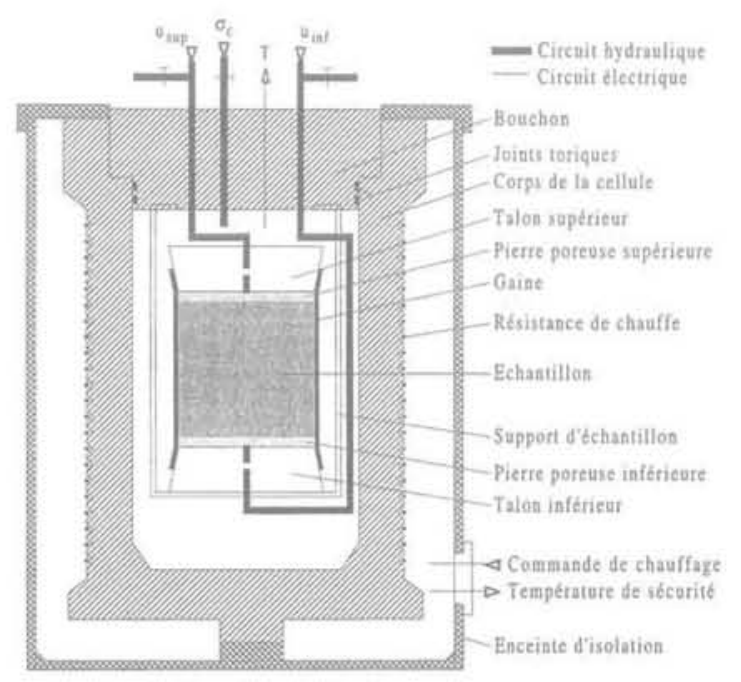

FIQ.1 Coupe schématique de la cellule de perméabilité.

Schematic diagram of the permeability cell.

\section{Appareillage}

Dans sa configuration actuelle, l'appareillage est composé d'une cellule de perméabilité qui en constitue l'élément central et de systèmes de mise en confinement, de mise en pression d'eau différentielle, de chauffage et de régulation thermique. Les figures 1 à 4 présentent les coupes schématiques des installations décrites ci-après.

\section{1}

\section{La cellule de perméabilité}

Elle se présente sous la forme d'un cylindre rigide en acier inoxydable, auquel est associée une résistance électrique filiforme enroulée le long de sa face latérale externe, et de certains éléments qui en constituent la partie centrale nécessaire à la mise en place de l'éprouvette; l'ensemble est placé à l'intérieur d'une enceinte cylindrique pour l'isoler du milieu extérieur.

Cette cellule a la particularité de pouvoir supporter une contrainte isotrope de confinement de $20 \mathrm{MPa}$ sous une température de l'ordre de $200^{\circ} \mathrm{C}$ et de permettre de mesurer de très faibles débits d'eau de l'ordre de quelques $\mathrm{mm}^{3} / \mathrm{h}$. Elle est conçue pour recevoir des éprouvettes d'argile de $50 \mathrm{~mm}$ de diamètre, dont l'épaisseur peut atteindre jusqu'a $50 \mathrm{~mm}$.

Au cours d'un essai, l'éprouvette entourée latéralement d'une gaine de silastène résistant aux fortes températures est placée entre deux talons métalliques légèrement coniques, rigides et inoxydables. Des pierres poreuses sont disposées entre l'éprouvette et les talons, pour faciliter l'écoulement de l'eau interstitielle dans le circuit de drainage et empêcher les particules argileuses fines de s'y infiltrer. Le circuit de drainage est constitué de tubes capillaires en acier inoxydable et de robinets. Les tubes sont reliés aux pierres poreuses à travers de passages transversant les talons dans leurs axes. Les robinets sont disposés en amont de certains éléments pour les isoler du système et en aval d'autres pour leur mise à l'air ou leur purge éventuelle. Une feuille d'aluminium est intercalée entre la gaine et l'éprouvette, pour répartir de façon homogène la température à l'intérieur et autour de cette dernière et, par conséquent, assurer son conditionnement. La gaine est fixée sur les talons au moyen d'une colle spéciale caractérisée par une bonne tenue aux fortes températures et une bonne résistance à l'eau, pour empêcher toute infiltration possible du fluide de confinement dans l'éprouvette. Le fluide de confinement utilisé est une huile connue pour sa bonne tenue aux fortes températures. L'ensemble est immergé dans I'huile au moyen d'un support dont les montants sont fixés sur le bouchon de la cellule, qui est lui-même vissé sur le corps de cette dernière. Des joints toriques en assurent l'étanchéité.

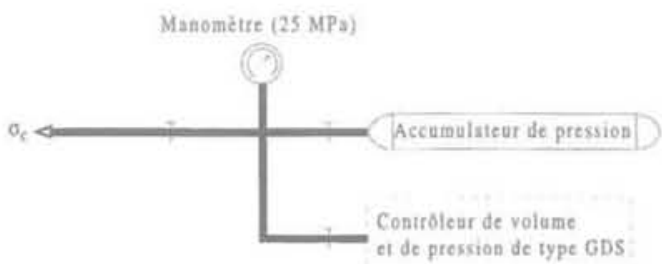

FG. 2 Coupe schématique du système de mise en confinement.

Schematic diagram of the confining system. 


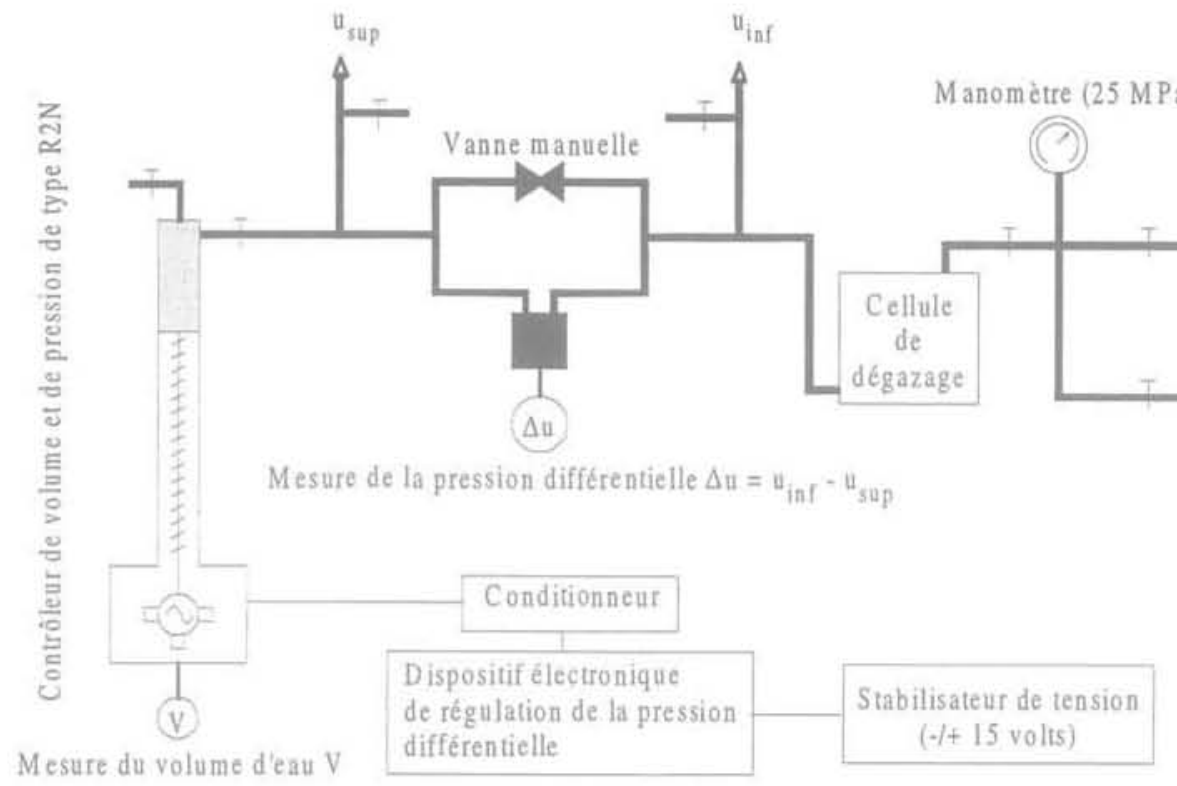

Accumulateur de pression

Contrôleur de volume et de pression de type GDS

Circuit hydraulique

- Circuit électrique

FG. 3 Coupe schématique du système de mise en pression d'eau différentielle. Schematic diagram of the differential pore water pressure system.

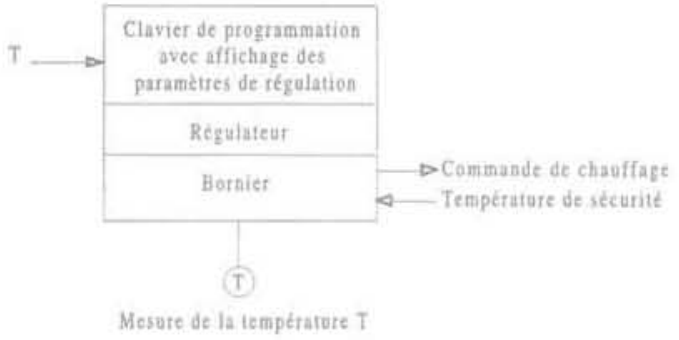

FIG. 4 Coupe schématique du système de chauffage et de régulation thermique. Schematic diagram of the heating and thermal regulation system.

\section{Le système de mise en confinement}

Il permet d'appliquer à l'éprouvette, par l'intermédiaire du fluide remplissant la cellule (l'huile), une contrainte isotrope de confinement $\sigma_{c}$ au moyen d'un contrôleur de volume et de pression, de réguler cette contrainte au moyen d'un accumulateur hydro-pneumatique de pression rempli d'eau et gonflé à l'azote et de contrôler sa valeur au moyen d'un manomètre à cadran de $25 \mathrm{MPa}$ de capacité placé entre l'accumulateur et la cellule de perméabilité.

\section{Le système de mise en pression différentielle}

Il permet d'exercer sur l'éprouvette, par l'intermédiaire du circuit de drainage, une pression d'eau différentielle $\Delta u$ résultant de l'application :

- d'une pression d'eau $u_{\text {inr }}$ sur la face inférieure au moyen d'un contrôleur de volume et de pression et sa régulation au moyen d'un accumulateur hydro-pneumatique de pression, via une cellule de dégazage remplie d'eau désaérée, avec contrôle visuel continu au moyen d'un manomètre à cadran de $2,5 \mathrm{MPa}$ de capacité :

- d'une pression d'eau $u_{\text {sup }}$ sur la face supérieure et sa régulation au moyen d'un contrôleur de volume et de pression de sorte que la pression différentielle $\Delta \mathrm{u}=\mathrm{u}_{\mathrm{nt}}$ - $u_{\text {sup' }}$ préfixée par l'expérimentateur, demeure constante tout au long de l'essai.

Ce système comporte en outre une vanne manuelle qui permet, lorsqu'elle est ouverte, de mettre en communication les faces inférieure et supérieure de l'éprouvette et d'y équilibrer les pressions d'eau $\left(\mathrm{u}_{\mathrm{inr}}=\right.$ $\mathrm{u}_{\text {sup }}$ ). La pression interstitielle résultante étant la même en tout point de l'éprouvette, celle-ci est alors soumise à une « contre-pression isotrope $»$.

La régulation de la pression différentielle s'effectue par le biais d'un dispositif électronique approprié, alimenté en courant stabilisé au moyen d'un stabilisateur de tension via un onduleur qui lui assure une protection totale contre les coupures de courant et les variations brusques de tension éventuelles. Un potentiomètre associé à ce dispositif permet, lorsque la vanne manuelle est fermée, d'établir directement une différence de pression entre les faces inférieure et supé- 
rieure de l'éprouvette. La pression interstitielle résultante est différente d'une section à l'autre de l'éprouvette, qui est donc soumise à un gradient hydraulique dans son axe.

\section{4}

\section{Le système de chauffage et de régulation thermique}

Il permet d'appliquer à l'éprouvette, par le biais de l'élément chauffant (résistance électrique), une montée ou une descente en température jusqu'à une valeur égale à la température T préfixée par l'expérimentateur et de l'asservir à cette valeur au moyen d'un régulateur autoadaptatif, appartenant à la génération des régulateurs mono-boucle à microprocesseur. La montée en température peut s'effectuer soit en continu, soit par paliers successifs, avec une vitesse comprise entre 10 et $15^{\circ} \mathrm{C} / \mathrm{h}$. La baisse de température s'effectue uniquement par le flux de fuite de la cellule de perméabilité. La constante de temps du système de régulation thermique est ainsi fixée par la vitesse de descente en température, qui est une donnée résultant de la construction de la cellule (volume de confinement et isolation thermique). Cette constante de temps thermique est de l'ordre de $3,5^{\circ} \mathrm{C} / \mathrm{h}$ à $70^{\circ} \mathrm{C}$.

Ce système, dont l'alimentation électrique est assurée vỉa l'onduleur utilisé pour le système de mise en pression hydraulique, comporte en outre un système de sécurité thermique qui interrompt le chauffage dès que la température mesurée dépasse la température de service du dispositif expérimental. Cette dernière peut être définie sur une échelle comprise entre 0 et $400^{\circ} \mathrm{C}$ mais, pour des raisons de sécurité de l'appareillage, elle a été limitée à $200^{\circ} \mathrm{C}$.

\section{5}

\section{Les appareils de mesure}

La mesure de la pression différentielle $\Delta u$ appliquée à l'éprouvette s'effectue au moyen d'un capteur différentiel de $0,7 \mathrm{MPa}$ de capacité, auquel est adjoint un amplificateur à faible bruit assurant son alimentation et son équilibrage. La valeur mesurée de cette pression est affichée à l'aide d'un multimètre à affichage numérique. La tension de sortie est calibrée de façon à ce qu'une pression de $100 \mathrm{kPa}$ corresponde à une valeur de 1 volt sur le multimètre.

La mesure du volume d'eau V sortant de l'éprouvette s'effectue par mesure du déplacement du piston du contrôleur de volume et de pression, utilisé pour la mise en contre-pression sur la face supérieure de l'éprouvette. Le piston, dont la course maximale est de $20 \mathrm{~cm}$ pour un volume maximal de liquide à déplacer de $200 \mathrm{~cm}^{3}$, est commandé par une vis à billes de pas égal à $1 \mathrm{~mm}$. La vitesse maximale de rotation de la vis à billes est de $14 \mathrm{tr} / \mathrm{min}$. La tension de sortie, comprise entre 0 et 10 volts, pour le volume d'eau déplacé est engendrée par une roue codeuse reliée à un convertisseur analogique-numérique, qui est lui-même relié au dispositif électronique utilisé pour réguler la pression différentielle. Sa précision est de 0,2 volt.

La mesure de la température $\mathrm{T}$ au niveau de l'éprouvette s'effectue au moyen d'un thermocouple de type
( $\mathrm{K})$ d'une classe de précision de l'ordre de $\pm 1^{\circ} \mathrm{C}$ à $25^{\circ} \mathrm{C}$ de température ambiante, qui délivre des mesures comprises entre 0 et $600^{\circ} \mathrm{C}$.

\section{6}

\section{L'acquisition des données}

Au cours d'un essai, les signaux émis par les appareils de mesure sont transmis au système d'acquisition des mesures constitué d'un multiplexeur de voies et relié à un micro-ordinateur. Ce système permet d'effectuer des enregistrements automatiques des paramètres mesurés à des cadences temporelles préfixées par l'expérimentateur. Le micro-ordinateur assure la gestion des données brutes, c'est-à-dire l'affichage de ces dernières sur l'écran sous forme de courbes en fonction du temps et leur saisie en mémoire de masse au moyen d'un logiciel du commerce.

\section{3}

\section{Le matériau d'essai}

Il s'agit d'une éprouvette de $50 \mathrm{~mm}$ de diamètre et $50 \mathrm{~mm}$ d'épaisseur découpée dans une galette d'argile prélevée en front de taille à $30 \mathrm{~m}$ de profondeur en carrière souterraine sur le site de Provins, puis conservée dans une chambre humide à $85 \%$ d'humidité et régulée à la température de $6^{\circ} \mathrm{C}$.

L'argile, constituée presque exclusivement de kaolinite, présente les caractéristiques physiques moyennes suivantes (Rousset, 1988) :

$\begin{array}{ll}\text { - densité humide : } & \gamma=21,4 \mathrm{kN} / \mathrm{m}^{3}, \\ \text { - densité des grains : } & \gamma_{\mathrm{s}}=27 \mathrm{kN} / \mathrm{m}^{3}, \\ \text { - teneur en eau : } & \mathrm{W}=16,9 \% \\ \text { - porosité : } & \mathrm{n}=0,32, \\ \text { - degré de saturation : } & \mathrm{S}_{\mathrm{r}}=97 \text { à } 100 \% .\end{array}$

On notera une teneur en eau élevée et une porosité faible, caractéristiques d'une argile plastique peu perméable.

\section{4}

\section{Programme expérimental et procédure d'essais}

La présente étude a pour but essentiel, d'une part, de vérifier le bon fonctionnement global de l'appareillage et, d'autre part, de valider une méthode de détermination du coefficient de perméabilité des argiles sous contrainte et température:

- la vérification du fonctionnement de l'appareillage porte sur :

- la régulation de la température,

- la régulation de la pression différentielle,

- la réqulation de la pression de confinement,

- la réponse des systèmes de mise en confinement et en pression d'eau différentielle aux variations de température ;

- la méthode expérimentale mise en œuvre pour déterminer le coefficient de perméabilité du matériau d'essai est schématisée par le mode opératoire représenté sur la fïgure 5. 


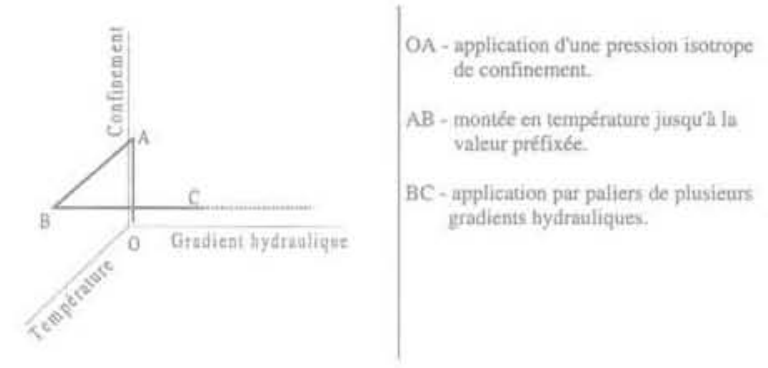

FG.5 Mode opératoire de l'essai de perméabilité sous contrainte et temperature.

Procedure of the permeability test under stress and temperature.

Cette méthode est basée sur la mesure en fonction du temps de la quantité d'eau qui traverse l'éprouvette soumise à un gradient de pression interstitielle résultant de l'application à une température donnée de la pression différentielle. L'opération peut être répétée pour plusieurs valeurs du gradient de pression interstitielle et plusieurs températures dans la limite possible de fonctionnement du dispositif expérimental (capacité de la cellule, sensibilité des appareils de mesure). Un débit d'eau est calculé pour chacune des opérations réalisées et une valeur du coefficient de perméabilité du matériau est déduite.

\section{1}

\section{Programme expérimental exécuté}

Il comporte un certain nombre d'essais répartis en trois séries correspondant chacune à une température donnée. Les essais sont identifiés par les valeurs préfixées de la pression différentielle $\Delta u$ et de la température T (tableau I).
La saturation du circuit de drainage est une opération qui consiste à remplir les tubes capillaires du système de mise en pression différentielle avec de l'eau distillée et à les purger par la suite afin de chasser les bulles d'air éventuellement présentes (l'argile étant caractérisée par un degré de saturation voisin de $100 \%$, sa saturation préalable n'a pas été jugée indispensable) ;

- les opérations réalisées à température constante à partir de cet état initial consistent à faire varier dans chaque série d'essais le gradient de pression interstitielle par paliers de quelques jours et à mesurer en fonction du temps le volume d'eau drainé de l'éprouvette, ainsi que les valeurs de pression différentielle et de température effectivement appliquées. Au cours de ces phases, la pression d'eau appliquée sur la face inférieure de l'éprouvette a été ramenée à 0,4 $\mathrm{MPa}$. La đistribution des paliers a été définie de manière croissante pour une série d'essais et décroissante pour la série suivante afin que, d'une part, l'incrément de charge induit par le passage d'une étape de chargement à l'autre soit faíble et si possible identique pour tous les essais et, d'autre part, la durée nécessaire à son application soit relativement très courte par rapport au temps consacré aux mesures du volume d'eau sortant de l'éprouvette.

\section{5}

\section{Résultats des essais et analyse}

Les résultats détaillés des mesures sont présentés dans l'annexe A1. L'analyse de ces résultats, dont la liste récapitulative est donnée dans le tableau II, conduit aux observations faites ci-après sur la sensibilité des éléments qui composent l'appareillage et leurs performances.

tabieaul Liste des essais. Tests list.

\begin{tabular}{|c|c|c|c|c|c|c|c|c|c|c|c|c|c|}
\hline Serrie & \multicolumn{5}{|c|}{1} & \multicolumn{4}{|c|}{2} & \multicolumn{4}{|c|}{3} \\
\hline$N^{0}$ & 01 & 02 & 03 & 04 & 05 & 06 & 07 & 08 & 09 & 10 & 11 & 12 & 13 \\
\hline $\mathrm{T}\left({ }^{\circ} \mathrm{C}\right)$ & \multicolumn{5}{|c|}{20} & \multicolumn{4}{|c|}{80} & \multicolumn{4}{|c|}{130} \\
\hline$\Delta u(\mathrm{kPa})$ & 50 & 60 & 80 & 100 & 200 & 200 & 150 & 100 & 50 & 50 & 100 & 150 & 200 \\
\hline
\end{tabular}

\section{2}

\section{Procédure d'essai suivie}

Elle comporte une phase initiale commune à tous les essais et une phase spécifique à chacun d'entre eux : - les opérations réalisés au cours de la phase initiale sont les suivantes :

- montage de l'éprouvette dans la cellule,

- saturation du circuit de drainage,

- application d'une pression d'eau isotrope de $0,2 \mathrm{MPa}$, $5 \mathrm{MPa}$,

application d'une pression de confinement de

- réglage du système de chauffage à la température de $20^{\circ} \mathrm{C}$. heures.
- stabilisation de l'ensemble pendant quelques

\section{1}

\section{Mesure de la température}

L'écart enregistré entre la consigne T et la mesure $T_{m}$ est très faible. Il l'est d'autant plus que la température est élevée (voir encadré ci-après). Cet écart peut être annulé en corrigeant l'équation d'étalonnage du thermocouple au moyen des coefficients correcteurs définis sur la droite de la figure 6 .

\begin{tabular}{|c|c|c|c|c|}
\hline $\mathrm{T}_{\mathrm{e}}$ & $\begin{array}{l}T_{\operatorname{mig}} \\
\left.{ }^{\circ} \mathrm{C}\right)\end{array}$ & $\begin{array}{l}T_{\text {mate }} \\
\left({ }^{\circ} \mathrm{C}\right)\end{array}$ & $\mathrm{T}_{\mathrm{ni}}$ & $\begin{array}{c}\Delta \mathrm{T}=\mathrm{T}_{\mathrm{M}}-\mathrm{T}_{\mathrm{c}} \\
\mathrm{l}^{\circ} \mathrm{C}\end{array}$ \\
\hline 20 & 21,889 & 22,348 & 22,110 & $+2,110$ \\
\hline 80 & 81,791 & 81,807 & 81,786 & $+1,786$ \\
\hline 130 & 131,687 & 131,858 & 131,754 & $+1,754$ \\
\hline
\end{tabular}




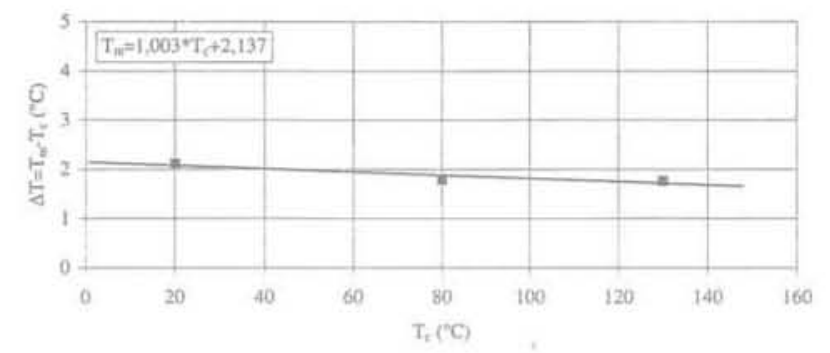

FG.6 Droite de correction de la fonction d'étalonnage du thermocouple. Catibration of the thermocouple.
L'écart type et à peu près constant à une température donnée, mais croît légèrement avec celle-ci (voir encadré ci-après). Le coefficient de variation est extrêmement faible. Il l'est d'autant plus que la température est élevée, ce qui correspond à une faible dispersion des mesures. Ceci constitue une preuve de la bonne qualité de régulation de la température.

\begin{tabular}{l|c|c|c}
\hline Température moyenne $\left({ }^{\circ} \mathrm{C}\right)$ & 22,110 & 81,786 & 131,754 \\
\hline Écart type $\left({ }^{\circ} \mathrm{C}\right)$ & 0,381 & 0,422 & 0,465 \\
\hline Coefficient de variation $(\%)$ & 1,7 & 0,5 & 0,4 \\
\hline
\end{tabular}

TABLEAUII Récapitulatif des résultats d'essais. Surnmary of tests results.

\begin{tabular}{|c|c|c|c|c|c|c|c|c|c|c|c|}
\hline \multirow[b]{2}{*}{$\mathrm{N}^{\circ}$} & \multirow[b]{2}{*}{$T_{0}\left({ }^{\circ} \mathrm{C}\right)$} & \multirow[b]{2}{*}{$\Delta u_{c}(\mathrm{KPa})$} & \multicolumn{3}{|c|}{$T_{m}\left({ }^{\circ} \mathrm{C}\right)$} & \multicolumn{3}{|c|}{$\Delta u_{m}(\mathrm{RPa})$} & \multicolumn{3}{|c|}{$Q_{m}\left(m m^{3} / h\right)$} \\
\hline & & & $m_{4}$ & $\sigma_{+}$ & $G_{i}$ & $\mathrm{~m}_{\mathrm{du}}$ & $\sigma_{A 4}$ & $\mathrm{C}_{\text {ain }}$ & $m_{0}$ & $\sigma_{1 y}$ & $C_{4}$ \\
\hline 01 & \multirow{5}{*}{20} & 50 & 22,349 & 0,262 & 1,2 & 55,316 & 2,000 & 3,6 & 2,286 & 0,293 & 12,8 \\
\hline 02 & & 60 & 22,063 & 0,452 & 2,0 & 64,452 & 2,117 & 3,3 & 2,511 & 0,207 & 8,3 \\
\hline 03 & & 80 & 22,097 & 0,474 & 2,1 & 88,535 & 1,603 & 1,8 & 3,422 & 0,225 & 6,6 \\
\hline 04 & & 100 & 21,889 & 0,457 & 2,1 & 114,400 & 1.967 & 1,7 & 4,995 & 0,200 & 4,0 \\
\hline 05 & & 200 & 22,150 & 0,260 & 1,0 & 231,630 & 1,650 & 1,0 & 13,900 & 1,760 & 12,7 \\
\hline 06 & \multirow{4}{*}{80} & 200 & 81,791 & 0,414 & 0,5 & 231,902 & 1,343 & 0,6 & 17,103 & 1,662 & 9,7 \\
\hline 07 & & 150 & 81,807 & 0,445 & 0.5 & 171,784 & 2,111 & 1.2 & 11,614 & 0,724 & 6,2 \\
\hline 08 & & 100 & 81,759 & 0,407 & 0,5 & 118,574 & 1,365 & 1,2 & 8,682 & 0,848 & 9,8 \\
\hline 09 & & 50 & \multicolumn{9}{|c|}{ inexploitable } \\
\hline 10 & \multirow{4}{*}{130} & 50 & 131,737 & 0,485 & 0.4 & 55,159 & 1,823 & 3,3 & 18.884 & 1,474 & 7,8 \\
\hline 11 & & 100 & 131,687 & 0,338 & 0,3 & 112.943 & 2,278 & 2,0 & 42,238 & 4,682 & 11,1 \\
\hline 12 & & 150 & 131,734 & 0,483 & 0,4 & 168,932 & 2,556 & 1.5 & 72,713 & 3.288 & 4,5 \\
\hline 13 & & 200 & 131,858 & 0,554 & 0,4 & 225,550 & 1,547 & 0,7 & 93,243 & 4,647 & 5,0 \\
\hline
\end{tabular}

$\mathrm{X}$-paramètre désignant $\mathrm{T}$, $\Delta \mathrm{u}$ ou $\mathrm{Q}, \mathrm{X}_{\mathrm{c}}$-consigne, $\mathrm{X}_{\mathrm{m}}$ - mesure, $\mathrm{m}_{\mathrm{x}}$ - moyenne, $\sigma_{\mathrm{x}}$-ècart type, $\mathrm{C}_{\mathrm{x}}=100^{\circ} \sigma_{\mathrm{x}} / \mathrm{m}_{\mathrm{x}}$-coefficient de variation exprimé en $\%$.

\section{2}

\section{Mesure de la pression différentielle}

L'écart entre la consigne $\Delta \mathrm{u}_{\mathrm{f}}$ et la mesure $\Delta \mathrm{u}_{\mathrm{m}}$ est important. Cet écart est d'autant plus grand que le gradient de pression est élevé (voir encadré ci-après). Ceci peut s'expliquer par l'utilisation d'un potentiomètre à affichage numérique, mais à commande analogique, difficile à étalonner avec précision. La figure 7 présente les coefficients correcteurs à introduire dans l'équation d'étalonnage de la pression différentielle.

\begin{tabular}{|c|c|c|c|c|}
\hline$\frac{\Delta u^{2}}{(\mathrm{kPa})}$ & $\begin{array}{l}\Delta \mathrm{u} \\
(\mathrm{kPa})\end{array}$ & $\begin{array}{l}\Delta \mathrm{u}_{\text {(kix }} \\
(\mathrm{k} P \mathrm{~d})\end{array}$ & $\frac{\mathrm{Au}}{(\mathrm{kPa})}$ & $\Delta u=\underset{(k P a)}{\Delta u_{a}}-\Delta u_{c}$ \\
\hline 50 & 55,159 & 55,316 & 55,238 & $+5,238$ \\
\hline 100 & 112,913 & 118,740 & 115,306 & $+15,306$ \\
\hline 150 & 168,932 & 171,784 & 170,358 & $+20,358$ \\
\hline 200 & 225,550 & 231,902 & 229,694 & $+29,694$ \\
\hline
\end{tabular}




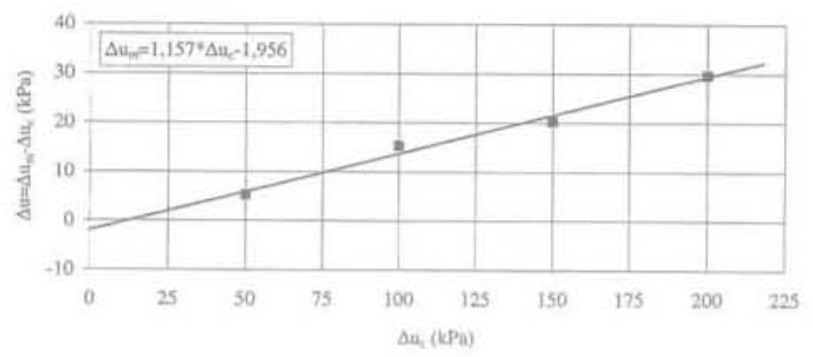

FG.7 Droite de correction de la fonction d'étalonnage du capteur de pression différentielle.

Calibration of the differential pore water pressure transducer.

Il est important de constater que l'écart type varie peu pour un gradient de pression donné et est d'autant plus faible que ce dernier est élevé (voir encadré ciaprès). Le coefficient de variation est très faible, ce qui constitue un gage de bonne précision pour le dispositif de régulation de la pression différentielle utilisé.

\begin{tabular}{l|c|r|r|r}
\hline $\begin{array}{l}\text { Gradient de pression } \\
\text { moyen }(\mathrm{kPa})\end{array}$ & 55,238 & 115,306 & 170,358 & 229,694 \\
\hline Écart type (kPa) & 1,912 & 1,870 & 2,334 & 1,513 \\
\hline $\begin{array}{l}\text { Coefficient } \\
\text { de variation (\%) }\end{array}$ & 3,5 & 1,6 & 1,4 & 0,8 \\
\hline
\end{tabular}

On notera que la pression d'eau $\mathrm{u}_{\mathrm{inf}}$ appliquée sur la face inférieure de l'éprouvette, qui est maintenue fixe dans tous les essais, semble croître légèrement avec la température, mais se stabilise au bout de quelques heures.

\section{3}

\section{Mesure du volume d'eau}

Les valeurs calculées du débit d'eau peuvent donner une idée sur le fonctionnement global du dispositif expérimental, sur l'interaction entre le système de chauffage et de régulation thermique et celui de mise en contre-pression et par conséquent sur leur influence sur les mesures du volume d'eau qui traverse l'éprouvette. Elles sont caractérisées par un écart type globalement faible, qui semble augmenter avec la température mais sans relation apparente avec le gradient de pression interstitielle (tableau II). Cependant, leur coefficient de variation apparaît un peu plus élevé que ceux du gradient de pression interstitielle et de la température, mais varie dans des proportions acceptables. Ceci peut s'expliquer par la présence probable de bulles d'air dans le circuit de drainage, qui ont pour conséquence de modifier la trajectoire de l'écoulement de l'eau à travers les interstices de l'éprouvette et nécessitent une différence de pression importante pour les faire déplacer. On peut constater sur certaines courbes de variation du volume d'eau drainé en fonction du temps l'existence de paliers qui traduisent la tortuosité de l'écoulement. Mais, il paraît plus plausible que la raison principale soit le mode de régulation adopté au moyen d'un contrôleur de volume et de pression pour lequel il faut un volume suffisant d'eau pour que son moteur fasse un mouvement et davantage encore pour un tour complet. Ceci a pu être pallié par la saturation du circuit de drainage.

\section{4}

\section{Mesure de la pression de confinement}

La pression de confinement étant la même pour tous les essais, seuls des contrôles visuels ont été effectués de temps à autre au cours desquels on a pu constater une légère baisse, mais sur une période assez longue ( 2 à 3 semaines). Cette baisse peut être attribuée à la qualité de la régulation effectuée au moyen de l'accumulateur hydropneumatique utilisé. Elle s'est accentuée aux fortes températures, mais sans conséquence directe sur les résultats des mesures, d'autant plus que des réajustements ont été effectués à chaque fois qu'un écart significatif entre la consigne et la valeur lue sur le manomètre a été enregistré. Par contre, le gradient de pression interstitielle ne semble pas avoir d'influence sur la pression de confinement.

\section{5}

\section{Calcul du coefficient de perméabilité et interprétation}

Le coefficient de perméabilité $\mathrm{k}$, déterminé en régime permanent, est donné en fonction du gradient de pression interstitielle $\Delta \mathrm{u}$ appliqué à l'éprouvette à la température $\mathrm{T}$ au moyen de l'expression suivante :

$$
\mathrm{k}=\frac{\gamma_{w} \mathrm{~L}}{\mathrm{~S}} \frac{1}{\Delta \mathrm{u}} \frac{\sum_{i=1}^{i=m} \Delta \mathrm{V}_{i} \mathrm{t}_{\mathrm{i}}}{\sum_{i=1}^{i=m} \mathrm{t}_{i}^{2}}
$$

dans laquelle $\Delta V$ désigne la quantité d'eau, de poids volumique $\gamma_{\mathrm{w}}=10 \mathrm{kN} / \mathrm{m}^{3}$, correspondant à l'enregistrement i comptabilisé à l'instant $t$ depuis le début de l'essai et $\mathrm{m}$ le nombre de mesures effectuées, $\mathrm{S}=19,635 \mathrm{~cm}^{2}$ la section droite de l'éprouvette et $\mathrm{L}=50 \mathrm{~mm}$ son épaisseur.

L'annexe A2 présente les détails de calcul du coefficient de perméabilité. Le tableau III en donne les valeurs pour les gradients de pression différentielle et les températures appliqués à l'éprouvette.

Ces résultats appellent les remarques suivantes: - l'écart entre la consigne et la mesure, aussi grand soit-il, n'a aucune conséquence sur la fiabilité des mesures et leur représentativité dans la mesure où le calcul du coefficient de perméabilité s'effectue non pas en fonction des paramètres de consigne mais en fonction des paramètres mesurés, c'est-à-dire en fonction du gradient de pression interstitielle et de la température effectivement appliqués :

- quant aux mesures caractérisées par des coefficients de variation très faibles, elles sont très peu dispersées et peuvent être considérées comme excellentes compte tenu de l'échelle de variation probable du coefficient de perméabilité de l'argile en place entre $10^{-11}$ et $10^{-13} \mathrm{~m} / \mathrm{s}$;

- l'influence de la température sur le coefficient de perméabilité est considérable, celle du gradient de pression interstitielle peut être considérée comme négligeable. 
TABLEAUIII Valeurs du coefficient de perméabilité $\mathrm{k}$ de l'argile pour différentes valeurs de pression différentielle $\Delta \mathrm{u}$ et de température $T$ appliquées.

Values of the coefficient of permeability of clay for various applied values of pore water pressure $\Delta \mathrm{u}$ and temperature $\mathrm{T}$

\begin{tabular}{l|c|c|c|c}
$N$ & $T\left({ }^{\circ} \mathrm{C}\right)$ & $\Delta u(\mathrm{kPa})$ & $Q\left(\mathrm{~mm}^{\mathrm{T}} / \mathrm{h}\right)$ & $\mathrm{k}\left(\mathrm{x} 10^{-12} \mathrm{~m} / \mathrm{s}\right)$ \\
\hline 01 & 22,349 & 55,316 & 2,16 & 2,76 \\
\hline 02 & 22,063 & 64,452 & 2,43 & 2,66 \\
\hline 03 & 22,097 & 88,535 & 3,50 & 2,79 \\
\hline 04 & 21,889 & 114,400 & 4,89 & 3,02 \\
\hline 05 & 22,150 & 231,630 & 13,74 & 4,20 \\
\hline 06 & 81,791 & 231,902 & 16,73 & 5,10 \\
\hline 07 & 81,807 & 171,784 & 11,40 & 4,70 \\
\hline 08 & 81,759 & 118,574 & 8,42 & 5,02 \\
\hline 09 & - & - & - & - \\
\hline 10 & 131,737 & 55,159 & 19,25 & 24,69 \\
\hline 11 & 131,687 & 112,943 & 43,48 & 27,24 \\
\hline 12 & 131,734 & 168,932 & 71,39 & 29,89 \\
\hline 13 & 131,858 & 225,550 & 90,56 & 28,40 \\
\hline
\end{tabular}

Selon la loi de Darcy, le coefficient de perméabilité k est relié à la viscosité de l'eau $\eta$ par l'expression suivante:

$$
k=\frac{\bar{k}}{\eta} \gamma_{w} \text {, }
$$

où $\bar{k}$ désigne le coefficient de perméabilité intrinsèque du matériau et $\gamma_{w}$ le poids volumique de l'eau.

Dans l'hypothèse où $\vec{k}$ est constant, l'évolution du coefficient de perméabilité $k$ en fonction de la température T est donnée par l'expression suivante

$$
k(T)=k\left(T_{0}\right) \frac{\eta\left(T_{0}\right)}{\eta(T)},
$$

où $T_{0}$ désigne la température initiale.

La figure 8 donne l'évolution du rapport des viscosités $\log _{10}\left[\eta(T) / \eta\left(T_{0}\right)\right]$ en fonction de la température $T$. Cette évolution décrit une courbe qui semble vérifier la loi établie par Boisson et al. (1992) sur des roches argileuses au moyen de l'expression suivante :

$$
\log _{70} \frac{\eta(\mathrm{T})}{\eta\left(\mathrm{T}_{0}\right)}=\frac{1,3272\left(\mathrm{~T}_{0}-\mathrm{T}\right)-0,001053\left(\mathrm{~T}-\mathrm{T}_{\mathrm{D}}\right)^{2}}{\mathrm{~T}+105} \text {, }
$$

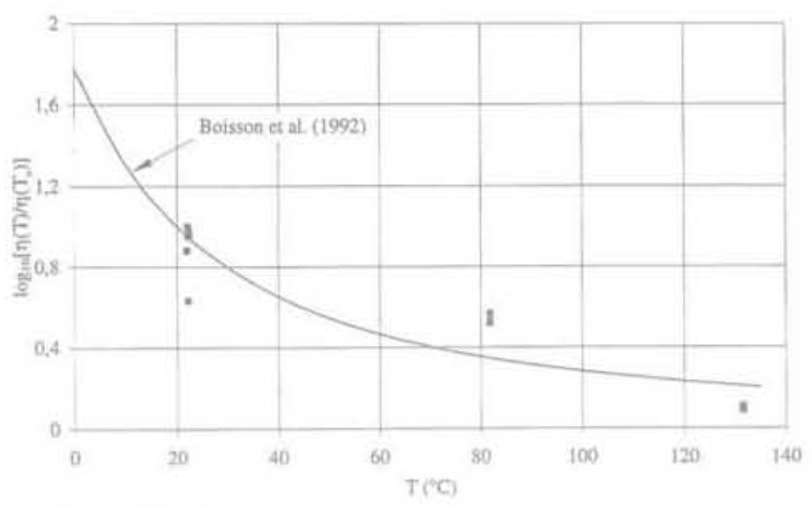

FG.8 Loi de variation de la viscosité de l'eau interstitielle en fonction de la température. Variation of the pore water viscosity as a function of temperature.

où $\mathrm{T}_{0}=20^{\circ} \mathrm{C}$.

On notera une diminution importante de la viscosité de l'eau interstitielle avec la température. Cette diminution est traduite par une forte augmentation du coefficient de perméabilité de l'argile.

\section{6 \\ Conclusion}

On notera la bonne qualité de régulation de l'ensemble du dispositif expérimental, la fiabilité des mesures et leur représentativité. On doit, toutefois, se poser la question suivante : à défaut de donner des valeurs représentatives du coefficient de perméabilité de l'argile en place, ce dispositif surestime-t-il les mesures ou plutôt les sous-estime-t-il ? Il est encore trop tôt pour répondre à cette question, et il faudra donc amasser un grand nombre de résultats sur différents types d'argiles pour en tirer une conclusion. Il faudra aussi garder à l'esprit que la loi de Darcy, qui constitue la base de la description de l'écoulement de l'eau dans les pores de l'argile, n'est pas toujours en mesure de décrire de manière satisfaisante les propriétés hydrauliques des sols en place. La variabilité naturelle du matériau peut constituer un obstacle majeur à une évaluation correcte et précise de son coefficient de perméabilité car, à l'échelle du massif, il peut exister des cheminements préférentiels qui augmentent considérablement la perméabilité d'ensemble par rapport à ce que l'on peut mesurer sur de petites éprouvettes en laboratoire.

Les résultats obtenus mettent en évidence l'influence de la température sur la perméabilité de l'argile et montrent qu'elle n'est pas beaucoup influencée par le gradient de pression interstitielle. Dans la gamme des gra-

\section{Bibliographie}

Al-Tabaa A., Wood D.M. - Some measurements of the permeability of Kaolin. Geotechnique, 1987, vol. 37 , n० 4, pp. 499-503.

Boisson J.Y., Billiotte J., Norotte V. - Etude au laboratoire de l'imfluence de la température sur le fluage des roches argileuses. Rapport final, Communauté européenne de l'Énergie atomique, $n^{\circ} \mathrm{F}$ 1 W 0163 F. 1992

Leroueil S., Lerat P., Hight D.W., Powell J.M. - Hydraulic conduction of a recent estuarine silty clay at Bothkennar. Geotechnique, 1992, vol. 42, n² 2, pp. 275-288

Little J.A. Wood D.M. Paul M.A. Bouazza A. - Some laboratory measurements of permeability of Bothkennar clay in rela tion to soil fabric. Geotechnique. 1992. vol, $42, n^{\circ} 2,355-361$.

Olsen H.W Nichols R.W. Rice T.L - Low gradient permeability measurements in a triaxial system. Geotechnique, 1985 vol. $35, n^{\circ} 2$, pp. $145-157$.
Read M.D., Meredith P.G., Murrell S.A. Permeability measurement techniques under hvdrostatic and deviatoric stress conditions. Rock and Great depth, Maury et Fourmaintraux ed. Balkema, Rotterdam, 1989, pp, 211-217.

Rousset G. - Comportement mécanique des argiles profondes. Application au stockage des déchets radioactifs. Thèse de doctorat de I'ENPC, 1988 
dients hydrauliques étudiés ici, on peut noter que le coefficient de perméabilité déterminé à $130^{\circ} \mathrm{C}$ est 5 à 6 fois supérieur à celui déterminé à $80^{\circ} \mathrm{C}$ qui est, lui-

\section{REMERCIEMENTS}

L'étude a été réalisée en partie pour l'Agence nationale pour la gestion des déchets radioactif (ANDRA). L'auteur remercie B. Bazargan-Sabet. même, de 1,5 à 2 fois supérieur à celui déterminé à $20^{\circ} \mathrm{C}$. Ces variations peuvent être attribuées à des variations de la viscosité de l'eau interstitielle avec la température.

S. Chanchole et D. Théval pour leur contribution à certaines parties de cette étude, notamment lors de la mise au point de l'appareillage.

\section{Annexe 1. \\ Résultats des mesures}
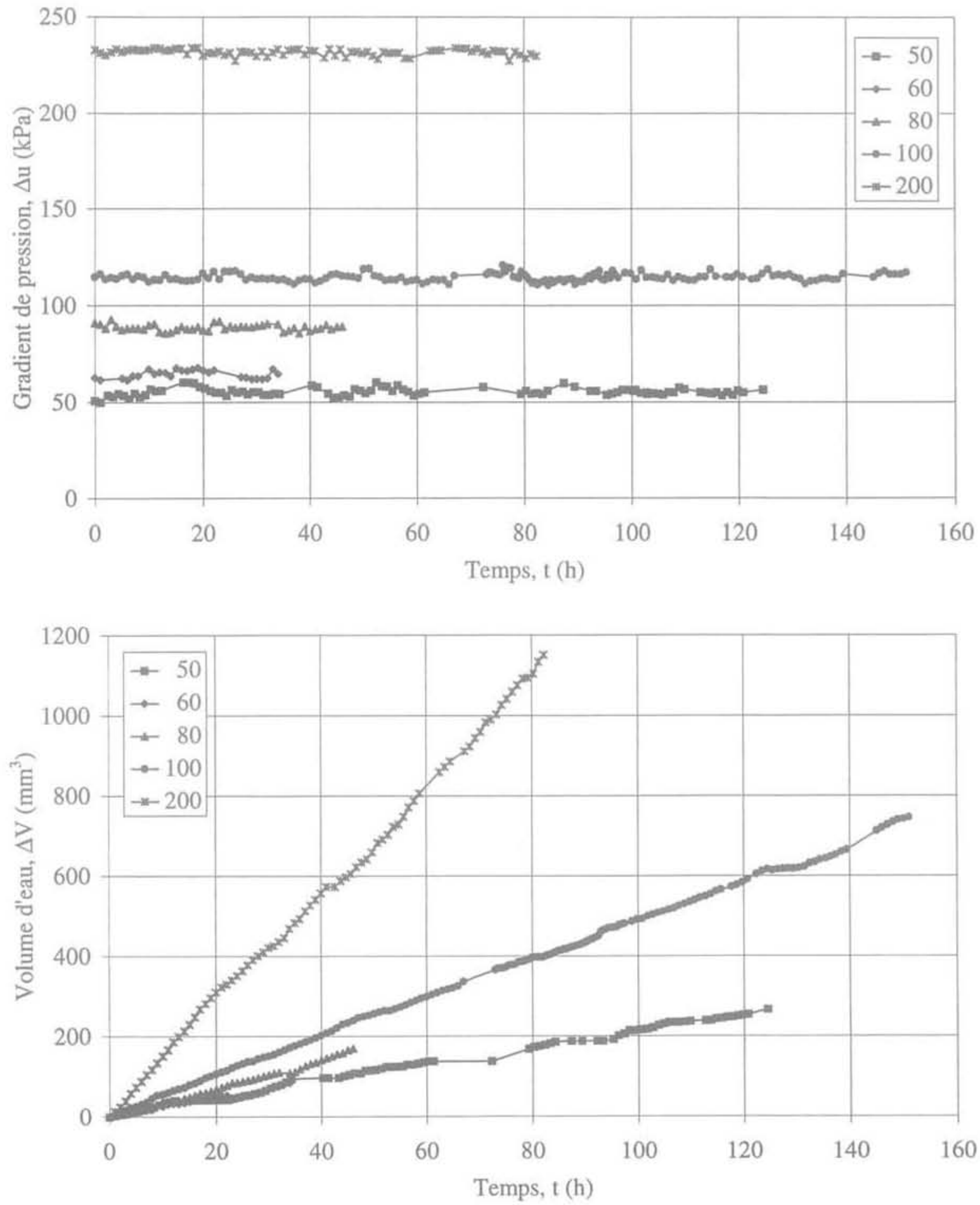

FIG. 1AT Évolution du débit d'eau à la température $\mathrm{T}=20^{\circ} \mathrm{C}$ pour les gradients hydrauliques $\Delta \mathrm{u}_{\mathrm{c}}=50,60,80,100$ et $200 \mathrm{kPa}$ considérés.

Volumetric flow rate versus time for temperature $\mathrm{T}=20^{\circ} \mathrm{C}$ at hydraulic gradients $\Delta \mathrm{u}_{\mathrm{c}}=50,60,80,100$ and $200 \mathrm{kPa}$. 

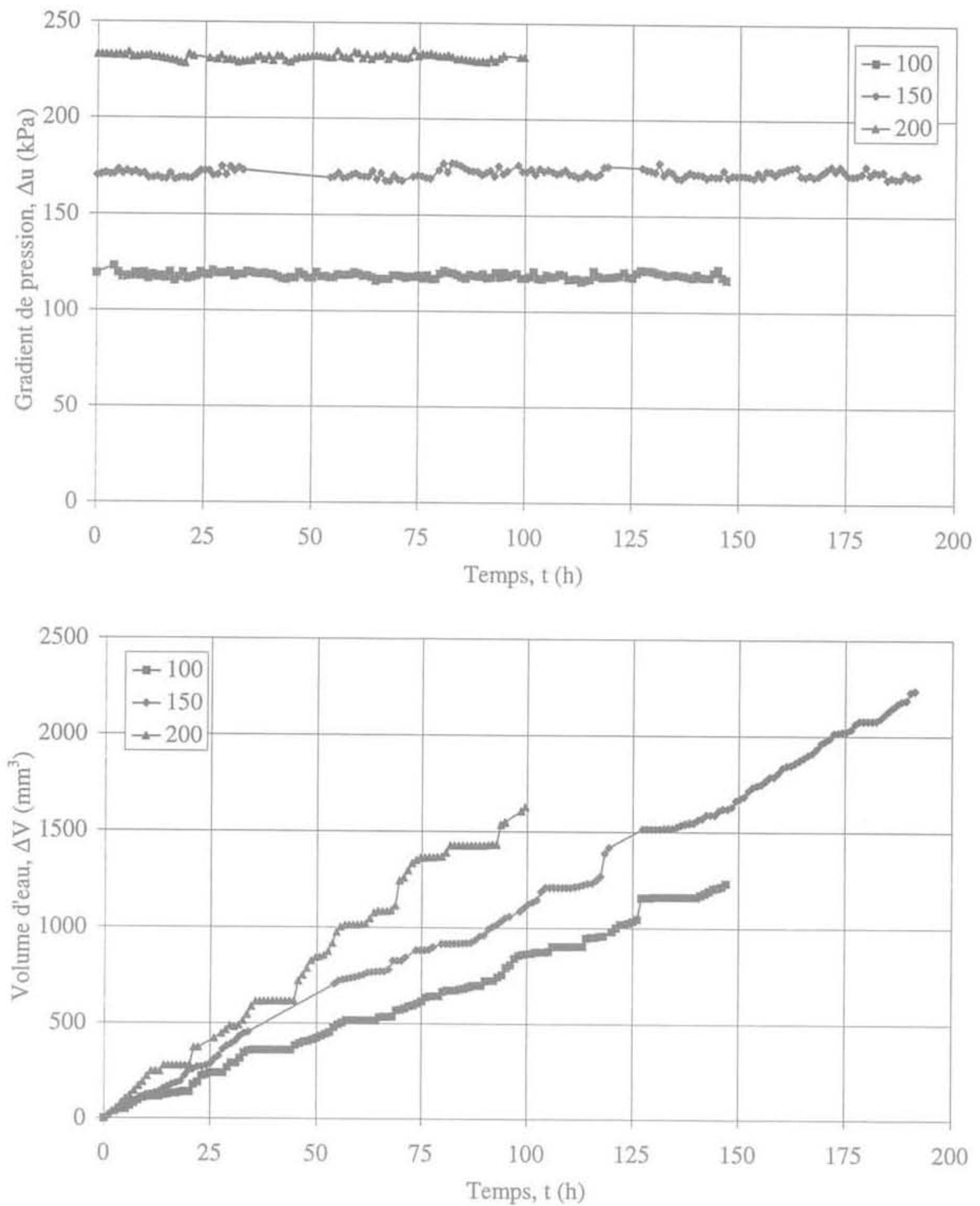

FG. 1 A2 Évolution du débit d'eau à la température $\mathrm{T}=80^{\circ} \mathrm{C}$ pour les gradients hydrauliques $\Delta \mathrm{u}_{\mathrm{c}}=100,150 \mathrm{et}$ $200 \mathrm{kPa}$ considérés.

Volumetric flow rate versus time for temperature $T=80^{\circ} \mathrm{C}$ at hydraulic gradients $\Delta \mathrm{u}_{\mathrm{f}}=100,150$ and $200 \mathrm{kPa}$. 

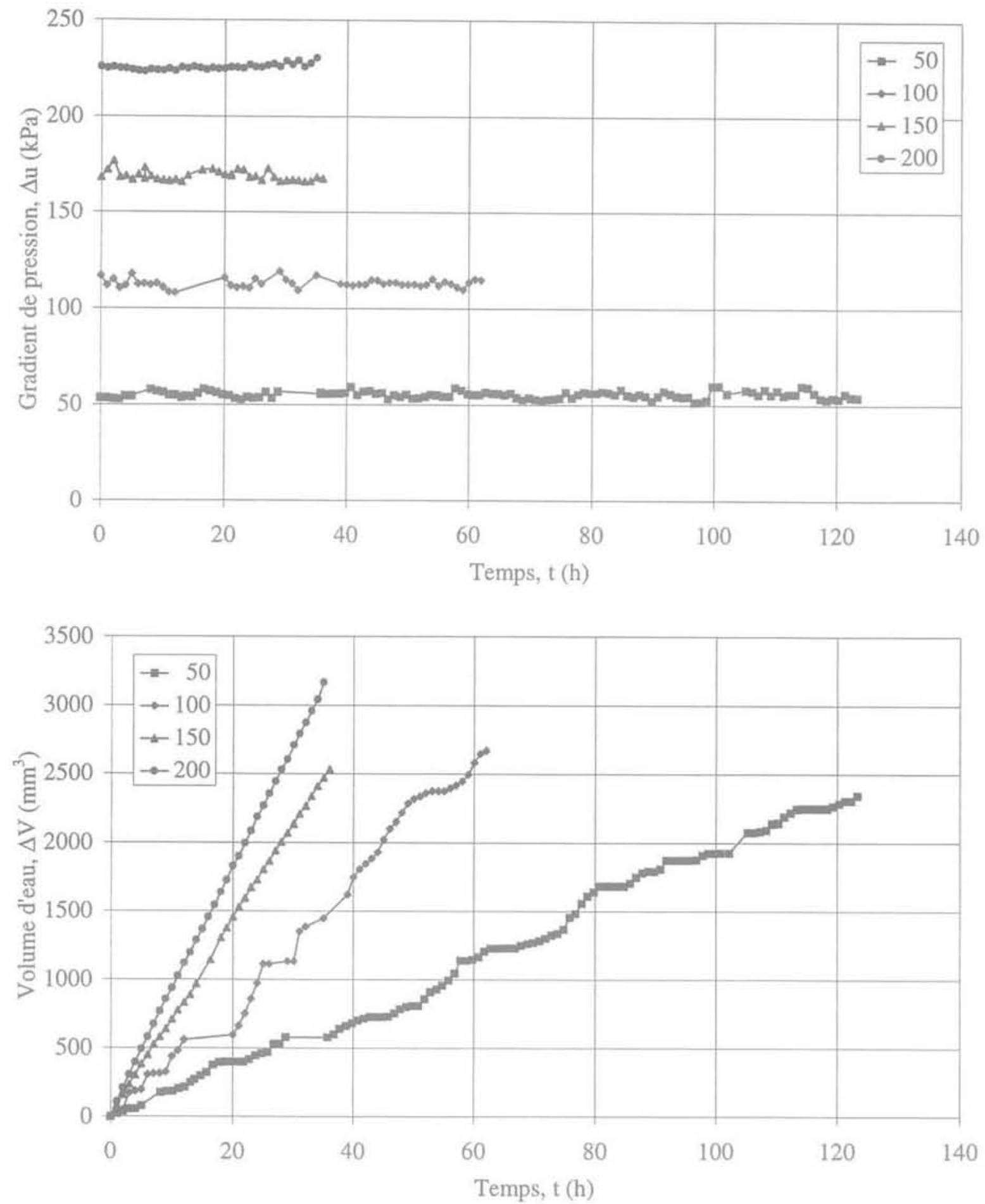

FiG. 1A3. Éyolution du débit d'eau à la température $\mathrm{T}=130^{\circ} \mathrm{C}$ pour les gradients hydrauliques $\Delta \mathrm{u}_{\mathrm{c}}=50,100,150 \mathrm{et}$ 200 kPa considérés.

Volumetric flow rate versus time for temperature $T=130^{\circ} \mathrm{C}$ at hydraulic gradients $\Delta u_{c}=50,100,150$ and $200 \mathrm{kPa}$. 


\section{Annexe 2.}

\section{Calcul analytiques}

L'interprétation des résultats d'essais repose essentiellement sur l'exploitation des mesures du volume d'eau traversant l'éprouvette effectuées en fonction du temps. L'examen des courbes correspondantes permet de constater que, dans la gamme de gradients hydrauliques étudiés ici, il est possible de relier la vitesse d'écoulement $v$ de l'eau sortant de l'éprouvette au gradient hydraulique i auquel elle est soumise par l'expression suivante :

$$
\mathrm{v}=\mathrm{ki} \text {, }
$$

qui n'est rien d'autre que la loi de Darcy, dans laquelle $k$ désigne le coefficient de perméabilité du matériau. Dans cette relation:

- la vitesse d'écoulement est calculée au moyen de l'expression :

$$
\mathrm{v}=\mathrm{Q} / \mathrm{S} \text {, }
$$

dans laquelle $\mathrm{S}=19,635 \mathrm{~cm}^{2}$ désigne la section droite de l'éprouvette;

- le gradient hydraulique est relié à la pression différentielle par l'expression:

$$
\mathrm{i}=\Delta \mathrm{u} / \gamma_{\mathrm{w}} \mathrm{L} \text {, }
$$

dans laquelle $\mathrm{L}=50 \mathrm{~mm}$ désigne l'épaisseur de l'éprou- vette et $\gamma_{\mathrm{w}}=10 \mathrm{kN} / \mathrm{m}^{3}$ le poids volumique de l'eau. D'où l'expression suivante du coefficient de perméabilité :

$$
\mathrm{k}=\frac{\gamma_{\mathrm{w}} \mathrm{L}}{\mathrm{S}} \frac{\mathrm{Q}}{\Delta \mathrm{u}} \text {. }
$$

Le débit $Q$ est déterminé par minimisation de la fonction :

$$
F(Q)=\sum_{i=1}^{\text {inth }}\left(\Delta V_{i}-Q t_{1}\right)^{2},
$$

dans laquelle $\Delta \mathrm{V}$ désigne la quantité d'eau correspondant à l'enregistrement i comptabilisée à l'instant t depuis le début de l'essai et $\mathrm{m}$ le nombre de mesures effectuées.

La dérivée de $\mathrm{F}$ par rapport à Q égalée à zéro donne:

d'où finalement :

$$
Q=\frac{\sum_{i=1}^{l=m} \Delta V_{i} t_{i}}{\sum_{i=1}^{i=m} t_{i}^{2}},
$$

$$
\mathrm{k}=\frac{\gamma_{\mathrm{w}} \mathrm{L}}{\mathrm{S}} \frac{1}{\Delta \mathrm{u}} \frac{\sum_{i=1}^{i=m} \Delta \mathrm{V}_{\mathrm{i}} \mathrm{t}_{\mathrm{i}}}{\sum_{\mathrm{i}=1}^{i=m} \mathrm{t}_{i}^{2}} .
$$

